\title{
Migration of Hem-o-lok clip into the common hepatic duct after laparoscopic bile duct exploration: A case report
}

\author{
Zhenhua Tan ${ }^{(1)}$ | Renrui Wan | Hai Qian | Ping Xie
}

Department of Hepatobiliary Surgery, Huzhou Central Hospital, Zhejiang University Huzhou Hospital, Affiliated Central Hospital of Huzhou Teachers

College, Huzhou, China

\section{Correspondence}

Renrui Wan, Department of

Hepatobiliary Surgery, Huzhou Central Hospital, Zhejiang University Huzhou Hospital, Affiliated Central Hospital of Huzhou Teachers College. 1558 North Third Ring Road, Huzhou 313000, China.

Email: barry985@163.com

\section{Funding information}

Huzhou Public Welfare General Project, Grant/Award Number: 2019GYB08 and 2020GY17

\begin{abstract}
Hem-o-lok clip migration into the bile duct can lead to stone formation and granulation tissue hyperplasia. This report discusses a case wherein four clips migrated into the bile duct after laparoscopic bile duct exploration.
\end{abstract}

\section{K E Y W O R D S}

clip migration, common bile duct stone, Hem-o-lok clip, laparoscopic bile duct exploration, laparoscopic cholecystectomy

\section{1 | INTRODUCTION}

Laparoscopic cholecystectomy and laparoscopic bile duct exploration are the main treatment options for gallbladder and biliary tract diseases. In laparoscopic surgery, Hemo-lok clips are widely used, and Hem-o-lok clip migration into the bile duct can lead to stone formation and granulation tissue hyperplasia. This report discusses a case wherein four clips migrated into the bile duct after laparoscopic bile duct exploration. The patient successfully underwent laparoscopy and choledocholithotomy.

Laparoscopic cholecystectomy (LC) and laparoscopic bile duct exploration (LCBDE) are the main treatment options for gallbladder and biliary tract diseases. With the development of laparoscopic surgery, many types of surgical clips have been used during these procedures. As of 2018, approximately 100 cases of post-cholecystectomy clip migration (PCCM) have been reported. ${ }^{1}$ To date, the mechanism of PCCM has not been clearly understood. This report discusses the case of clip migration in a 72-year-old woman; four clips migrated into the bile duct after laparoscopic bile duct exploration. We extracted four Hem-o-lok clips from the common hepatic duct and found that there were two possible ways by which clip migration occurred.

\section{2 | CASE PRESENTATION}

A 72-year-old woman presented with repeated right upper abdominal pain for 1 month. She had undergone LC and LCBDE 4 years ago because of cholecystitis with gallbladder stone and common bile duct (CBD) stones. We ligated the cystic duct and gallbladder arteries separately using 
two Hem-o-lok clips. Two weeks later, the patient was discharged when the result of T-tube radiography revealed no abnormalities. After 4 months, the patient was hospitalized again with upper abdominal pain for 10 days. She underwent ultrasound and magnetic resonance cholangiopancreatography (MRCP) examination, and both imaging modalities showed hilar bile duct stones and CBD dilation. The laboratory indices were as follows: DBS, $19.7 \mu \mathrm{mol} / \mathrm{L}$ (normal <6.8 $\mu \mathrm{mol} / \mathrm{L}$ ); ALT, $132.6 \mathrm{U} / \mathrm{L}$ (nor$\mathrm{mal}<40.0 \mathrm{U} / \mathrm{L}$ ); and AST, 85.3 U/L (normal <35.0 U/L). CBD stones were treated with endoscopic retrograde cholangiopancreatography (ERCP) 6 days later, and the patient was readmitted to our department 4 years later. Upon physical examination, her sclera and skin were not icteric; there was tenderness on the right upper quadrant without rigidity or rebound tenderness. MRCP and ultrasound revealed hilar bile duct stones and intrahepatic and extrahepatic bile duct dilation (Figures 1 and 2). We performed laparoscopy and choledocholithotomy as treatment. During the surgery, we noted that the Hem-o-lok clips used in the previous surgery were not in their original position and had migrated into the CBD (Figure 3). Moreover, we observed two Hem-o-lok clips among the stones (Figure 4, white arrow and white arrowhead). After removing them, the left hepatic duct was visible, whereas the right hepatic duct remained obscured by some tissues. We excised some soft tissues for pathological examination to rule out malignant tumors, and the examination indicated purulent inflammation of the tissue. We eventually removed another two Hem-o-lok clips from the right side of the hepatic bile duct; their removal was difficult because the clips had firmly adhered to the surrounding tissues. Unlike the first two clips, these clips had residual soft tissues (Figure 4, black arrow). The intrahepatic bile duct and CBD were examined again using choledochoscopy, and no obvious stones and foreign bodies were found. We inserted a 20-Fr T-tube for drainage. One week later, $\mathrm{T}$-tube radiography findings were normal. Thus, the patient was discharged within the next few days.

\section{DISCUSSION}

Hem-o-lok clips have been widely used in LC and LCBDE in recent years. However, they can migrate into the bile duct and cause stone formation and granulation tissue hyperplasia, which can occlude the bile duct, thereby leading to impaired liver function and obstructive jaundice. Clinical manifestations include abdominal pain, fever, nausea, jaundice, and vomiting. Severe cases may be complicated by purulent cholangitis, septic shock, or even death. The patient in this case underwent LC and LCBDE at our hospital 4 years ago, and CBD stones recurred 4 months later. When the stone was removed by ERCP, there was no evidence of Hem-o-lok clip migration into the bile duct. Unlike metallic titanium, the Hem-olok clip had similar density as the stones on both CT and MRI. The Hem-o-lok clips in this patient gradually migrated from the abdominal cavity into the bile duct after 4 years; in previous reports, the median time of PCCM was 26 months (range: 11 days to 20 years). ${ }^{2}$ However, the exact mechanism of PCCM remains unclear. Based on previous reports and our own experience, the following six hypotheses were formulated:

1. Surgical clips may migrate into the bile duct via the T-tube sinus (because it is part of the wall of the $\mathrm{T}$-tube sinus) or into the T-tube sinus after T-tube removal (we believe that the clips on the left side of the hepatic duct might have migrated in this way).
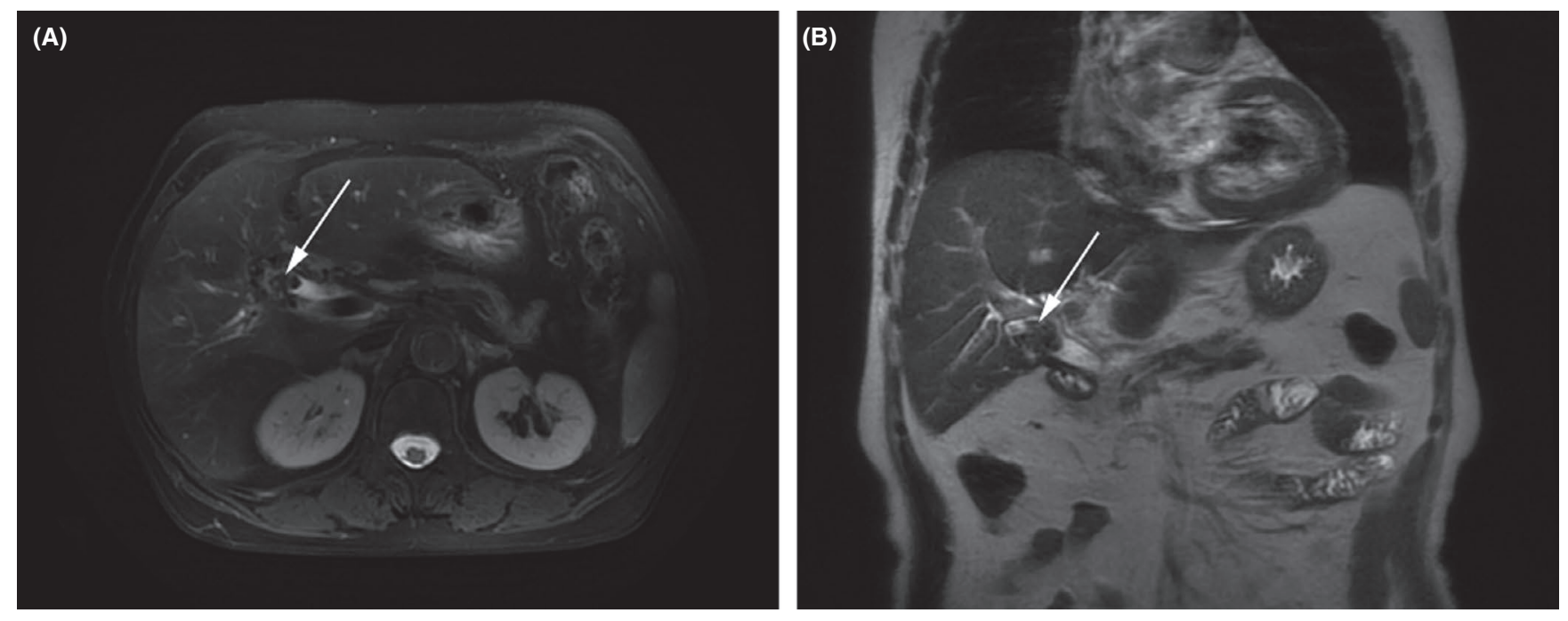

F I G URE 1 MRI showing bile duct dilatation with low signal mass in the hepatic portal bile duct (white arrow) 

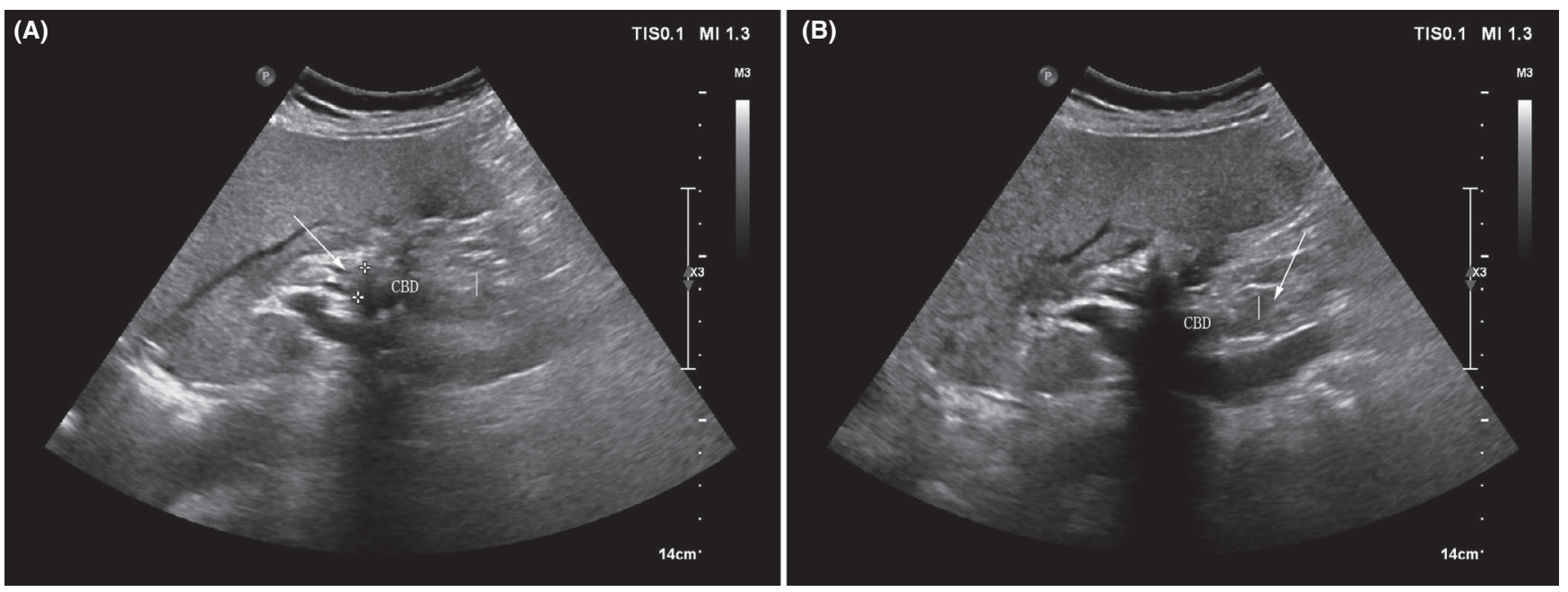

F I G URE 2 Ultrasonography showing a hyperechoic mass with bile duct dilatation in the extrahepatic bile duct (white arrow)

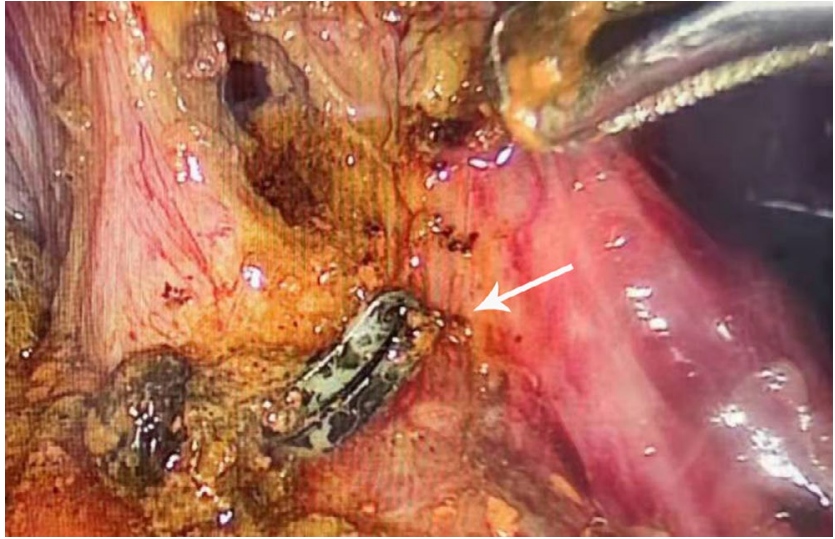

F I G U RE 3 One Hem-o-lok clip was removed from the common bile duct (white arrow)

2. The junction of the cystic duct is mostly anatomically flared. If we ligate the cystic duct too close to the CBD, the ligated cystic duct and surgical clip may retract into the biliary tract under the pressure from the surrounding tissues after losing the traction of the gallbladder (the clips in the right side of the hepatic duct might have migrated in this way).

3. Others hypothesized that inflammatory rejection response to the surgical clips (foreign body) near the wall of the bile duct is also a possibility. ${ }^{3}$

4. If the surgical clips are placed near the entrance of the T-tube to the bile duct, they will be pushed into the lumen of the CBD by the surrounding structures after the T-tube is removed.

5. Raoul et al. suggested that surgical clips might be applied improperly, and the incomplete closure of cystic duct wounds can lead to the development of biloma, which could be drained into the CBD through the cystic duct. ${ }^{4}$

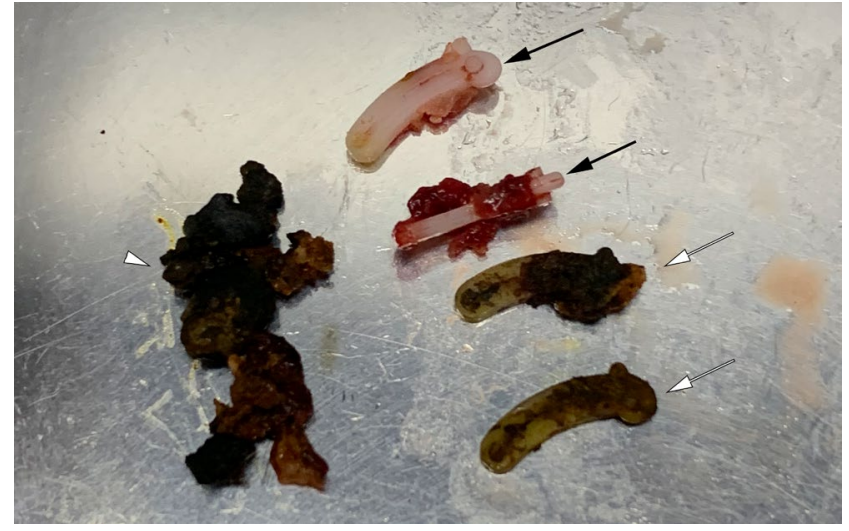

F I G U RE 4 Extraction of two Hem-o-lok clips encased in stones (white arrows), another two clips (black arrows) with softtissue residues, necrotic tissue, and stones blocking the common hepatic duct (white arrowhead)

6. Localized inflammatory processes may lead to the erosion of the bile duct wall, which may result in the migration of surgical clips into the bile duct. ${ }^{5,6}$

How to reduce this phenomenon? Some reports recommend reducing the number of clips used in the cystic duct and gallbladder triangle or using both absorbable thread and clips to reduce the number of clips used. ${ }^{7-9}$ Other options to seal the cystic duct and artery without clips include harmonic scalpel and LigaSure. ${ }^{10-12}$ However, the safety of these methods has not been adequately verified. ${ }^{13}$ To reduce the risk of clip migration through the bile duct incision, we can make primary closure after $\mathrm{LCBDE}^{14}$ if the width of the CBD is more than $1 \mathrm{~cm}$. If there is no other choice besides non-absorbable clip, we should ligate the cystic duct $0.5-1.0 \mathrm{~cm}$ away from the CBD. It is best to ligate the gallbladder duct using absorbable sutures. 
When T-tube drainage is placed for the CBD, it is best to place the clips far from the T-tube.

\section{CONCLUSION}

The use of the non-absorbable clips has been proven safe and effective in most cases; however, the undesirable consequences caused by surgical clips migrating into the biliary tract or other body parts should be considered. In addition, the clip migration may be related to the individual's physique. Anyway, we should try to minimize the occurrence of ligation clip displacement.

\section{ACKNOWLEDGEMENTS}

This work was supported by the Huzhou Public Welfare General Project (2020GY17); and the Huzhou Public Welfare General Project (2019GYB08). We would like to thank Editage (www.editage.com) for English language editing. Published with written consent of the patient.

\section{CONFLICTS OF INTEREST}

The authors have no conflict of interest to disclose.

\section{AUTHOR CONTRIBUTIONS}

Zhenhua Tan collected the surgical data and wrote the manuscript. Renrui Wan conceptualized the study, collected the data, and designed the figure. Hai Qian and Ping Xie collected the data.

\section{CONSENT}

Written informed consent was obtained from the patient.

\section{ETHICAL APPROVAL}

The Ethics Committee of Huzhou Central Hospital approved this study (approved number: 20210729).

\section{DATA AVAILABILITY STATEMENT}

Data sharing is not applicable to this article as no datasets were generated or analyzed during the current study.

\section{ORCID}

Zhenhua Tan (D) https://orcid.org/0000-0003-1157-2509

\section{REFERENCES}

1. Rou WS, Joo JS, Kang SH, et al. Abdominal pain due to Hem-olok clip migration after laparoscopic cholecystectomy. Korean J Gastroenterol. 2018;72(6):313.
2. Hussameddin AM, Ibrahim AFI, Fahad AOR. Common bile duct stone formed around a migrated clip: an unexpected complication of laparoscopic cholecystectomy. Case Rep Gastrointest Med. 2018;2018:1-2.

3. Rawal KK. Migration of surgical clips into the common bile duct after laparoscopic cholecystectomy. Case Rep Gastroenterol. 2016;10(3):787-792.

4. Raoul JL, Bretagne JF, Siproudhis L, Heresbach D, Campion JP, Gosselin M. Cystic duct clip migration into the common bile duct: a complication of laparoscopic cholecystectomy treated by endoscopic biliary sphincterotomy. Gastrointest Endosc. 1992;38(5):608-611.

5. Ahn SI, Lee KY, Kim SJ, et al. Surgical clips found at the hepatic duct after laparoscopic cholecystectomy: a possible case of clip migration. Ann Surg Treat Research. 2005;15(5):279-282.

6. Schreuder AM, van Gulik TM, Rauws EAJ. Intrabiliary migrated clips and coils as a nidus for biliary stone formation: a rare complication following laparoscopic cholecystectomy. Case Rep Gastroenterol. 2019;12(3):686-691.

7. Yang CP, Cao JL, Yang RR, et al. Efficacy of electrocoagulation in sealing the cystic artery and cystic duct occluded with only one absorbable clip during laparoscopic cholecystectomy. $J$ Laparoendosc Adv Surg Tech Part A. 2014;24(2):72-76.

8. Feroci F, Lenzi E, Kröning KC, Scatizzi M. A single-institution review of the absorbable clips used in laparoscopic colorectal and gallbladder surgery: feasibility, safety, and effectiveness. Updates Surg. 2011;63(2):103-107.

9. Rohatgi A, Widdison AL. An audit of cystic duct closure in laparoscopic cholecystectomies. Surg Endosc. 2006;20(6):875-877.

10. Turial S, Engel V, Sultan T, Schier F. Closure of the cystic duct during laparoscopic cholecystectomy in children using the ligasure vessel sealing system. World J Surg. 2011;35(1):212-216.

11. Zanghì A, Cavallaro A, Di Mattia $\mathrm{P}$, et al. Laparoscopic cholecystectomy: ultrasonic energy versus monopolar electrosurgical energy. Eur Rev Med Pharmacol Sci. 2014;18:54-59.

12. Kandil T, El Nakeeb A, El Hefnawy E. Comparative study between clipless laparoscopic cholecystectomy by harmonic scalpel versus conventional method: a prospective randomized study. J Gastrointest Surg. 2010;14(2):323-328.

13. Abdallah E, Ellatif MA, Awady SE, et al. Is LigaSure a safe cystic duct sealer an ex vivo study. Asian J Surg. 2015(38):187-190.

14. Wu T, Xiong TG, Gao XZ, et al. Analysis of the efficacy and safety of primary suture after laparoscopic bile duct exploration and stone removal of common bile duct. J Hepatopancreatobiliary Surg. 2019;31(8):486-488.

How to cite this article: Tan Z, Wan R, Qian H, Xie P. Migration of Hem-o-lok clip into the common hepatic duct after laparoscopic bile duct exploration: A case report. Clin Case Rep. 2021;9:e04834. https://doi.org/10.1002/ccr3.4834 